\section{Malignant cell lines - why use APUDoma?}

SIR - Several investigators have referred to our cell lines, COLO 320 and 321 as APUDomas (meaning "containing amine precursor uptake and decarboxylase activities'). These cell lines were derived from a carcinoma of the colon and are interesting because of their replication of c-myc oncogene ${ }^{1}$. Our description ${ }^{2}$ of the cell lines included a list of cell products. The detection of several peptide hormones was subsequently interpreted by others as evidence that the cell line should be labelled "APUDoma".

Many diverse malignancies have been "frozen" in a stage of differentiation which results in activation of the genes for one or multiple peptide hormones ${ }^{3}$. There are probably malignant forms of every single kind of cell that could be labelled "APUDoma".

It seems reasonable that cell line designations should not be altered but that unique aspects of the cells should be noted if relevant to the work being described.

GEORGE E. MOORE

Department of Health and Hospitals,

Department of Surgical Oncology,

777 Bannock Street,

Denver, Colorado 80204-4507, USA

1. Alitalo, K. Schwab, M., Lin, C.C., Varmus, E.H. \& Bishop, M.J. Proc. natn. Acad. Sci. U.S.A. 80, $1707-$ 1711 (1983).

2. Quinn, I..A., Moore, G.E., Morgan, R.T. \& Woods, L. Cancer Res. 39, 4914-4924 (1983).

3. Stevens, R.E. \& Moore, G.E. Lancer, i, 118-119 (1983).

\section{Mechanism for heritability of intelligence}

SIR - I wish to suggest a partial answer to the important question of what mechanism in the central nervous system may account for the heritability of intelligence ${ }^{1}$.

Although we are far from having a generally agreed definition of intelligence, let alone an understanding of the mechanism responsible, I believe that even now we can specify one necessary neurophysiological aspect of the variation in degree of intelligence and show that it has an appreciable degree of genetic determination. Whatever intelligence is, it must require communication, by nerve impulses, among different regions of the brain. These impulses are transmitted along nerve fibres and across synapses with the participation of many different proteins (including enzymes) and other molecules, such as neurotransmitters (which are synthesized by specific enzymes). These 'transmission proteins', like all proteins, are composed of polypeptide chains which are direct gene products.

There is great genetic diversity among humans (within races); half or more of structural gene loci are polymorphic, that is, they have two or more common alleles (genes) at that locus ${ }^{2}$. We consequently expect appreciable genetic variability among individuals for the genes specifying these 'transmission proteins'. In addition to this direct genetic variation in the structure, and hence the activity of these proteins, there is a second kind of genetically specified variation: differences, among normal individuals, in the amount (concentration) of each specific protein. In particular, whenever enzyme concentrations, for example, dopamine- $\beta$. hydroxylase and catechol-O-methyltransferase (which, respectively, synthesize and metabolize the neurotransmitter noradrenaline) have been studied genetically, they have been found to be under appreciable genetic control ${ }^{3}$.

This genetic variability, both in structure and amount of transmission proteins is, of course, heritable from parent to offspring. This variability will affect nerve impulse transmission in the brain to some degree and in a variety of ways, such as in speed and accuracy. It therefore sets limits on information processing rates. Data from mice support this interpretation, directly showing heritability of nerve conduction velocity ${ }^{4,5}$ and synaptic transmission time ${ }^{6}$. This genetic control of information processing is, I submit, a sufficient basis for asserting that there is significant genetic determination of variation in human intelligence.

I do not suggest of course, that such a mechanism would operate in isolation. I believe that in all societies, environment (in the broadest sense, beginning prenatally) also has very important effects (positive and negative) on human intelligence.

\section{Departments of Zoology and \\ Anthropology, \\ University of Toronto,}

Toronto, Ontario, Canada M5S IAI

1. Maddox, J. Nature 309, 579 (1984)

. Nei, M. \& Roychoudhury, A.K. Evol. Biol 14, 1-59 (1982)

3. Weinshilboum, R.M. in Neurogenetics: Genetic Approaches to the Nervous System (ed. Breakefield X.O.) 257-282 (Elsevier, New York, 1979).

4. Hegmann, J.P., White, J.E., \& Kater, S.B. Behav. Genet. 31, 121-131 (1973).

Reed, T.E. Behav. Gener. 13, 257-265 (1983).

6. Reed, T.E. Behav. Genet. (in press).

\section{Atmospheric humidity in the nuclear winter}

SIR - Recent calculations ${ }^{1,2}$ and discussion of the possibility of a 'nuclear winter' consequent to widespread nuclear war may have ignored an important effect of atmospheric humidity. The nuclear winter is calculated ${ }^{2}$ to be severe for wars in the late spring or summer. In those seasons, at most assumed targets the dew point is quite high, usually no less than $55^{\circ} \mathrm{F}$ $\left(13^{\circ} \mathrm{C}\right)$. Soot from urban or forest fires would be produced intimately mixed in this humid air.

In order to reach the "nuclear winter" state this air is chilled. That portion of it which rises to the upper troposphere or higher reaches ambient temperatures when its rise stabilizes, in an hour or less; only on a much longer time scale does solar heating rewarm it. The remaining sooty air is below the warming influence of sunlight, and it cools to the low surface air temperatures calculated for the nuclear winter. In each case the air is cooled to a temperature at which the saturation water vapour pressure is far below the initial vapour content. The resulting condensate efficiently scavenges the soot aerosol, as in the precipitation cleaning of urban smog and the black rain observed after firestorms; precipitation removes both water and soot from the atmosphere.

Scavenging is unavoidable: in nuclear winter calculations the air begins warm and humid and ends cool and dry, a process which must efficiently purge it of its water vapour and aerosols. This effect has been ignored in the published calculation $\mathrm{s}^{1,2}$ because they begin by assuming a distribution of soot in the troposphere, and do not calculate condensation and scavenging. A quantitative understanding will require detailed calculation of rising sooty plumes and of condensation and scavenging both there and in the cooling troposphere.

Department of Physics,

JONATHAN KATZ

Washington University,

St Louis, Missouri 63130, USA

1. Turco, R.P., Toon, O.B., Ackerman, T., Pollack, J.B. \& Sagan, D. Science 222, 1283-1300 (1983).

2. Covey, C., Schneider, S. H. \& Thompson, S. L. Nature 308, $21-25$ (1984).

\section{DNA can be a selfish parasite}

SIR - Evans, recently pointed out a "fatal flaw" in all evolutionary discussions of selfish or parasitic DNA. The problem, as outlined by Evans, is that any element which imposes any cost on its host will be eliminated by natural selection; this selection pressure can be counteracted only by a mechanism for horizontal transfer (presumably virus-like infection of cells) or by a high rate of de novo generation of new elements. The latter condition is highly unlikely and the former would imply that parasitic DNA is merely a new label applicable only to conventional viruses. I cannot agree with these conclusions.

I have already explored ${ }^{2}$ in some detail the questions raised by Evans and the main conclusion is that parasitic DNAs can potentially spread very rapidly in natural populations despite the fact that they may also have significant negative effects on host fitness. A mechanism of horizontal spread is not necessary provided there is biparental inheritance of host genomes. This criterion is met in all sexually-reproducing, out-breeding higher eukaryotes. Parasitic elements capable of duplicate transposition are able to "colonize" new chromosomes at zygote formation. Thus, the elements can spread to new genomes by a means other than horizontal transfer. The general prediction of this model is that we expect a higher level of such parasites within the genomes of out-breeding higher 